\title{
Acute myocardial infarction as the first manifestation of paraganglioma
}

\author{
Sara Pinto, ${ }^{1}$ Raquel Ferreira, ${ }^{2}$ Anabela Gonzaga, ${ }^{2}$ José Mesquita Bastos ${ }^{2}$ \\ ${ }^{1}$ Internal Medicine Department; and ${ }^{2}$ Cardiology Department, Centro Hospitalar do Baixo Vouga, Aveiro, Portugal
}

\begin{abstract}
Paragangliomas (PGLs) are extra-adrenal neuroendocrine tumors, classified as sympathetic or parasympathetic according to their origin in the paraganglia of the autonomic nervous system. Sympathetic PGLs are mostly functional, presenting in a variable and non-specific way. We report a case of PGL, which was diagnosed further to an investigation of acute myocardial infarction in a postpartum woman, highlighting that the absence of typical symptoms may delay the diagnosis.
\end{abstract}

\section{Case Report}

A 41-year-old female was admitted to the Emergency Department (ED) of Centro Hospitalar of Baixo Vouga (CHBV) due to the sudden onset of intense and sharp chest pain that irradiated to the left arm, with a two-hour duration, without other complaints. Her past medical history was significant for migraine headaches, but with no cardiovascular risk factors such as arterial hypertension, diabetes, dyslipidemia or smoking. She had delivered her fourth child one week before and had

Correspondence: Sara Barbosa Pinto, Internal Medicine Department, Centro Hospitalar do Baixo Vouga, Avenida Doutor Artur Ravara, 3810-193 Aveiro, Portugal. Tel.: +35.1234378300. E-mail: pintosara88@gmail.com

Key words: Paraganglioma; acute myocardial infarction; catecholamines.

Contributions: SP designed and wrote the manuscript with support of RF and AG; JMB revised the manuscript and contributed to intellectual content; all authors gave final approval of the version to be submitted and any revised version.

Conflict of interests: the authors declare no conflict of interests.

Further information: the authors declare that the patient included in the manuscript received enough information and gave her written informed consent to participate in this study. This manuscript did not receive any type of funding.

Received for publication: 11 November 2018.

Revision received: 13 February 2019.

Accepted for publication: 14 February 2019.

This work is licensed under a Creative Commons Attribution NonCommercial 4.0 License (CC BY-NC 4.0).

(C) Copyright S. Pinto et al., 2019

Licensee PAGEPress, Italy

Italian Journal of Medicine 2019; 13:124-127

doi:10.4081/itjm.2019.1115 no history of complications of any of the gestations and/or deliveries. There were no abnormal findings on physical examination. The initial electrocardiogram (ECG) showed sinus tachycardia (heart rate $100 \mathrm{bpm}$ ) with a slight ST-segment elevation in inferior (DII, DIII, AVF) and V4-V5 leads, and ST depression with T wave inversion in DI-AVL and V1-V3 leads. A bedside transthoracic echocardiogram was performed and revealed medial inferolateral and apical lateral akinesia, extending to medial anterolateral, medial inferior and apical inferior areas. Analytical workup presented an elevation of myocardial necrosis biomarkers $(\mathrm{MNb})$ : creatine phosphokinase $589 \mathrm{U} / \mathrm{L}$, troponin (cTnI) 7.6 $\mathrm{ng} / \mathrm{mL}$ and myoglobin $1859.6 \mathrm{ng} / \mathrm{mL}$. The patient was given low molecular weight heparin (30 mg IV), aspirin (300 $\mathrm{mg}$ po) and clopidogrel (600 $\mathrm{mg}$ po) and then transferred to a hemodynamic center, where a coronarography excluded coronary disease. A computed tomography angiography (CT-angiography) was then performed, which excluded the possibility of aortic dissection or pulmonary thromboembolism, but showed a $5 \times 2.1 \mathrm{~cm}$ nodular hypodense lesion in the right paravertebral region, with soft tissue density. The patient was transferred back to our institution where, apart from migraine headaches, she remained asymptomatic. The $\mathrm{MNb}$ gradually normalized, as did echocardiographic findings. Despite stress myocardiopathy being the most probable diagnosis, the patient was discharged medicated for an acute coronary syndrome (ACS), with an ACE inhibitor (lisinopril $5 \mathrm{mg}$ qd), a $\beta$-blocker (bisoprolol $2.5 \mathrm{mg} \mathrm{qd}$ ), double antiplatelet therapy (aspirin $100 \mathrm{mg}$ qd and clopidogrel $75 \mathrm{mg} \mathrm{qd}$ ), and a statin (atorvastatin $40 \mathrm{mg} \mathrm{qd}$ ).

Five days after being discharged, the patient returned to the ED of CHBV with chest pain and presenting again elevated $\mathrm{MNb}(\mathrm{cTnI} 3.15 \mathrm{ng} / \mathrm{mL}$ ), and ECG changes (ST depression in V2-V6 leads and T wave inversion in inferior leads). Physical examination was normal and a second ECG, performed later, 
was also normal. She was then admitted to the Cardiology ward for etiological study. While hospitalized the patient remained asymptomatic, hemodynamically stable and without chest pain. A cardiac magnetic resonance (cMRI) and a high-resolution chest CT (to further investigate the paravertebral mass) were performed. The cMRI revealed moderate hypokinesis of lateral medial-apical segments associated with delayed contrast enhancement and again mentioned a right paravertebral mass (Figure 1).

The high-resolution chest CT confirmed a nodular, soft tissue density, expansive lesion with $55 \times 23 \mathrm{~mm}$ apparently attached to the spine, admitting a neurogenic etiology, but not excluding a focus of extramedullary hematopoiesis or a lymphoma (Figure 2).

Given the suspicion of paraganglioma (PGL), laboratory tests of plasma and urine catecholamines and their metabolites were performed, showing an elevation of total plasma catecholamines and noradrenaline (Table 1). Chromogranin A (CgA) was measured and was within normal range $(2.5 \mathrm{nmol} / \mathrm{L})$ and an abdominal/pelvic CT excluded the presence of other mass lesions.

After these results it was considered that the patient had bouts of coronary vasospasm secondary to catecholamines release, and she was discharged medicated with antiplatelet monotherapy (aspirin $100 \mathrm{mg}$ qd), a calcium channel blocker (diltiazem $60 \mathrm{mg} 3 \mathrm{id}$ ), and oriented to Cardiology and Cardiothoracic Surgery outpatient consultation. A biopsy of the lesion was performed, and histological analysis was compatible with a ganglioneuroma. Video-assisted thoracoscopic surgery (VATS) was performed four months later; then a complete and uneventful excision of the tumor was achieved. The medication was stopped and, a year and a half after surgery, the patient remained

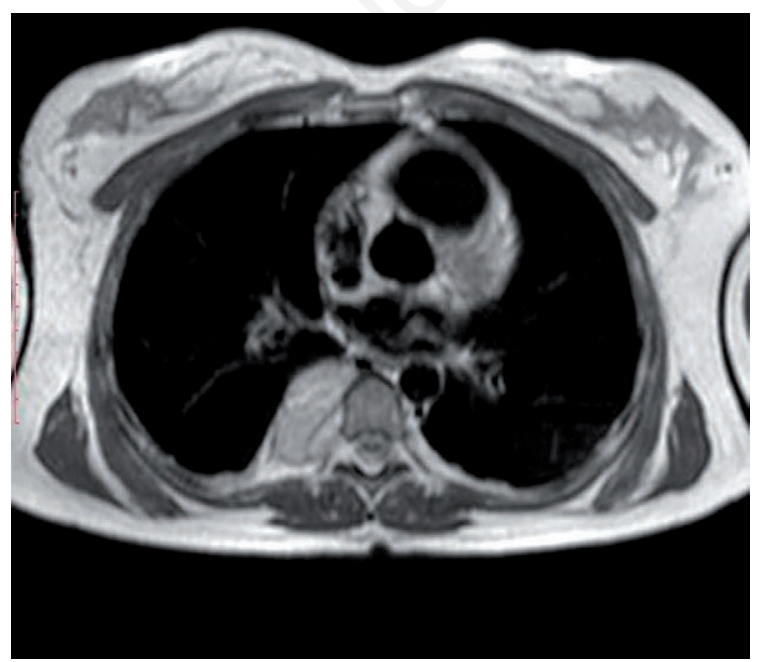

Figure 1. Cardiac magnetic resonance imaging showing a right paravertebral mass. asymptomatic, without recrudescence of chest pain or migraine, and without evidence of tumor recurrence.

\section{Discussion}

PGLs are extra-adrenal neuroendocrine tumors, classified as sympathetic or parasympathetic according to their origin in the paraganglia of the autonomic nervous system. In clinical practice, it is not uncommon that $P G L$ and pheochromocytoma are often referred to as the same pathological entity; however, the term pheochromocytoma is reserved to describe intra-adrenal PGLs. ${ }^{1}$ The sympathetic PGLs derive from chromaffin cells, and they occur from the skull to the pelvis, along the sympathetic chain. ${ }^{2}$ While PGLs preferentially secrete noradrenaline and normetanephrine, pheochromocytomas secrete adrenalin. ${ }^{3}$ Since the majority of sympathetic PGLs are functioning, they usually manifest with paroxysms resulting from episodic excess catecholamine release. Surprisingly, there is no consistent correlation between symptoms and circulating catecholamines levels. ${ }^{4}$ The main signs and symptoms of catecholamine ex-

Table 1. Assessment of plasma catecholamines and their metabolites.

\begin{tabular}{lcc}
\hline & Value & Reference value \\
\hline Adrenaline $(\mathrm{ng} / \mathrm{L})$ & 76 & $<84$ \\
\hline Noradrenaline $(\mathrm{ng} / \mathrm{L})$ & 496 & $<420$ \\
\hline Dopamine $(\mathrm{ng} / \mathrm{L})$ & 32 & $<94$ \\
\hline Total catecholamines $(\mathrm{ng} / \mathrm{L})$ & 604 & $<598$ \\
\hline Normetanephrine $(\mathrm{pg} / \mathrm{mL})$ & 13 & $<65$ \\
\hline Metanephrine $(\mathrm{pg} / \mathrm{mL})$ & 35 & $<196$ \\
\hline
\end{tabular}

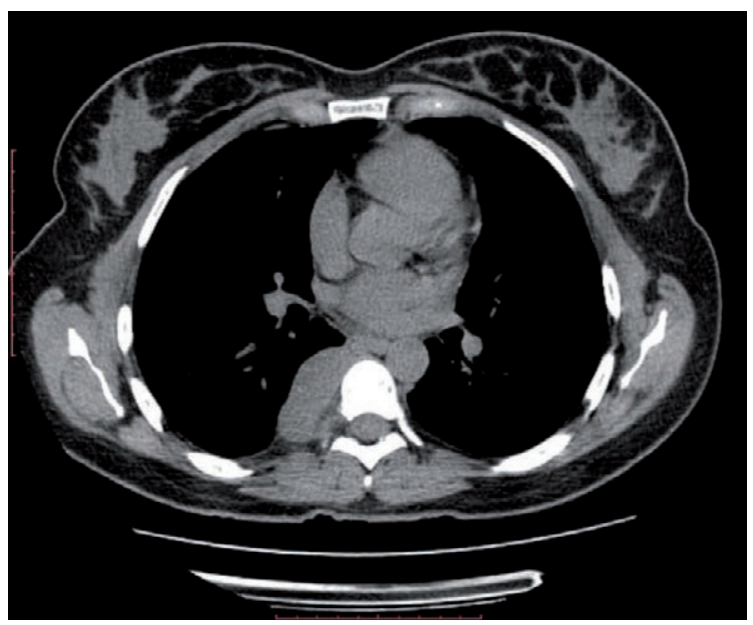

Figure 2. High-resolution chest computed tomography, which shows a nodular, soft tissue density lesion attached to the spine. 
cess include hypertension, palpitations, headaches and sweating. ${ }^{1}$ It is estimated that only half of patients with pheochromocytoma or paraganglioma have sustained hypertension and, the other half, paroxysmal hypertension or normotension. ${ }^{1}$ Tumors secreting norepinephrine are usually associated with sustained hypertension, whereas epinephrine and norepinephrine secreting tumors are associated with paroxysmal hypertension. ${ }^{4}$ Parasympathetic PGLs, on the other hand, are generally nonfunctional and present with compressive symptoms. ${ }^{5}$

Fortunately, an increasing number of paragangliomas are diagnosed before the onset of symptoms, either as an incidental finding on imaging, or by surveillance in subjects with known genetic susceptibility. ${ }^{4}$ PGLs can be sporadic or familial. Hereditary forms include familial paraganglioma syndrome, Von HippelLindau syndrome, multiple endocrine neoplasia type 2, neurofibromatosis type 1 and Carney-Stratakis dyad, requiring genetic tests for diagnosis in most cases. ${ }^{3}$

The diagnostic exams upon suspicion of a PGL, even in the absence of adrenergic symptomatology, include measurement of urine and plasma catecholamines and urine metanephrines (normetanephrine and metanephrine). ${ }^{6}$ The evaluation of $\mathrm{CgA}$ has been recommended, especially in patients with non-functioning PGLs. This test has a high sensitivity but low specificity, with false positive results occurring in the setting of renal insufficiency, hepatic insufficiency or proton pump inhibitor use, thus the interpretation of results should be careful. ${ }^{7}$

CT has a sensitivity and specificity of over $90 \%$ in locating adrenal pheochromocytomas but is less accurate in detecting extra-adrenal neuroendocrine tumors. On the other hand, T2-weighted MRI images are more sensitive for the detection of paragangliomas. Lastly, I-MIBG scintigraphy is considered the best image method to locate extra-adrenal tumors that are not recognized on CT or MRI. ${ }^{8}$ This is due to I-MIBG structural similarity to noradrenaline.

For its curative potential, elective surgery is the treatment of choice for PGLs. The exceptions to this are asymptomatic patients with non-functional and small-sized $(<2-3 \mathrm{~cm})$ tumors, where a more conservative approach can be considered. ${ }^{9}$

Only $15-30 \%$ of PGLs are malignant, and unless there is the presence of metastases in non-chromaffin tissue, no marker can differentiate a benign tumor from a malignant one. However, studies suggest that larger tumors, dopamine secretion, and elevated levels of $\mathrm{CgA}$ are associated with a greater malignant potential. ${ }^{10}$ Since the recurrence of PGLs can occur up to 20 years after presentation, the follow-up of these patients should be extended for life. ${ }^{3}$ In patients with functional PGLs, plasmatic metanephrines, urine metanephrines, and $\mathrm{CgA}$ levels should be monitored annually. $\mathrm{CgA}$ levels are especially important in those patients with normal metanephrine levels, being useful as a marker for the detection of recurrence and monitoring of response to different treatments. ${ }^{11}$

Imaging study (CT or MRI) should be performed yearly in patients with non-functional tumors. In patients with functional tumors it should be performed whenever there is a relevant change in biochemical markers. ${ }^{6}$

Although the incidence of acute myocardial infarction (AMI) in the obstetric population is low, it is rising due to advancing maternal age and medical comorbidities. ${ }^{12}$ Even though atherosclerotic disease is the major cause of AMI in this population, spontaneous coronary artery dissection (SCAD) is also a significant cause of AMI in ante, peri and postpartum periods. ${ }^{12}$ The pathogenesis of SCAD during these periods is unclear, with a probable role of physiological and hormonal changes contributing to an increased blood volume and altered anticoagulation mechanisms. ${ }^{13}$

The case presented reports a woman with an unclarified history of migraine, recently exposed to a relevant state of stress (delivery), who was diagnosed with an AMI without coronary disease. To exclude non-atherosclerotic coronary disease (as SCAD or pulmonary thromboembolism), a thoracic CT angiography was performed and incidentally revealed a nodular lesion in the right paravertebral region, initially assumed as an unrelated finding. Admitting, without certainty, an AMI diagnosis (stress myocardiopathy, for example, was not excluded), the patient was discharged medicated with an ACE inhibitor, a $\beta$ blocker, double antiplatelet therapy and a statin. After the patient returned a second time with chest pain, elevation of $\mathrm{MNb}$ and ECG changes suggestive of ischemia, it was mandatory to clarify the underlying cause. The cMRI, describing moderated hypokinesis of lateral medial-apical segments associated with delayed contrast enhancement, was fundamental to confirm the diagnosis of AMI.

Nevertheless, and despite there was no evidence of hypertension or other typical symptoms, the history of migraine, the recrudescence of cardiac complaints and the description/location of the paravertebral mass on CT, led to the hypothesis of neuroendocrine tumor. With high specificity when occurring, hypertensive paroxysms are less frequent in PGLs than in pheochromocytomas, which may justify its absence.

The measurement of catecholamine levels associated with biopsy findings, confirmed the diagnosis of ganglioneuroma, and at last clarified the etiology of these cardiac events. The biochemical workup, revealing only a slight elevation of total catecholamines and noradrenaline, without other findings would not be entirely elucidative. The sensitivity of these tests could have been increased by repeating them two or more times, especially following another eventual paroxysm, since a single measurement may not be suffi- 
cient, taking into account the highly fluctuating levels of catecholamines. Considering the risk of leading a catecholamine crisis, the performance of a biopsy in suspected cases of PGL is generally contraindicated. ${ }^{14}$ Although the high-resolution chest CT has raised the hypotheses of extramedullary hematopoiesis or lymphoma, a I-MIBG scintigraphy could have been useful in the diagnosis and less risky.

Some hypotheses try to explain the presence of angina and acute myocardial infarction in patients with neuroendocrine tumors: severe coronary spasm; direct myocardial damage by catecholamines and increased oxygen uptake due to secondary tachycardia. ${ }^{15}$ Several complications have been described associated with functional PGLs, including LV hypertrophy, ischemic heart disease, myocardial infarction, cardiac arrhythmias, Takotsubo cardiomyopathy, dilated cardiomyopathy, and shock. ${ }^{16}$ In this case, the PGL can explain both events: AMI of the inferior wall, due to the above reasons, and recrudescence of chest pain associated with elevation of $\mathrm{MNb}$ and dynamic electrocardiographic changes, by coronary spasm due to the paroxysmal release of catecholamines. Nevertheless, it is important to mention, while the etiology was not clarified, that the patient was treated with a $\beta$-blocker (bisoprolol) due the ACS. $\beta$-blockers should never be used before $\alpha$-adrenoceptor blockers, because of the risk hypertensive crisis. ${ }^{17}$

Despite questionable, after evaluation at a Cardiothoracic Surgery consult, taking into account the patient's own will and the usually benign nature of PGLs, it was considered that tumor excision was not urgent. The patient was submitted to surgery half a year later, without any intercurrence in the meanwhile. The surgical technique chosen was VATS, a type of thoracic surgery performed using a small video camera. Unless in cases of large tumors with a higher risk of malignancy, laparoscopic surgical resection is preferred for PGLs. ${ }^{1}$ During surgery, no mishaps were described. Preoperative drug therapy and intravascular volume expansion are two significant factors that improve the surgery prognosis. Currently, phenoxybenzamine is the most commonly used drug for the blockade of alpha-adrenergic receptors. ${ }^{3}$

The surgical resolution of PGL, made the patient not only asymptomatic, but also free from chronic medication. Despite this favorable clinical outcome, and since there is potential for recurrence, the patient maintains a scheduled follow-up.

\section{Conclusions}

PGLs are neuroendocrine tumors with a variable clinical presentation depending on their location and secretory profile. They may lead to emergency situations, and a prompt clinical suspicion is essential for an early diagnosis. In the absence of prior symptoms or elevated blood pressure, chest pain or even AMI, may be the initial manifestation. We highlight the importance of including PGLs in the differential diagnosis of AMI without coronary disease.

\section{References}

1. Chen H, Sippel RS, Pacak K. The NANETS Consensus Guideline for the diagnosis and management of neuroendocrine tumors: pheochromocytoma, paraganglioma \& medullary thyroid cancer. Pancreas 2010;39:775-83.

2. Lin D, Carty S, Young WF. Paragangliomas: epidemiology, clinical presentation, diagnosis, and histology. In: Post TW, ed. UpToDate. Waltham, MA: UpToDate Inc.; July 2018.

3. Palha A, Cortez L. Paragangliomas: diagnóstico, tratamento e seguimento. Rev Port Endocrinol Diabetes Metab 2017;12:215-22.

4. Clifton-Bligh R. Diagnosis of silent pheochromocytoma and paraganglioma. Exp Rev Endocrinol Metab 2013; 8:47-57.

5. Martins R, Bugalho MJ. Paragangliomas/pheochromocytomas: clinically oriented genetic testing. Int J Endocrinol 2014;794187.

6. Lenders JWM, Duh QY, Eisenhofer G, et al. Pheochromocytoma and paraganglioma: an Endocrine Society Clinical Practice Guideline. J Clin Endocrinol Metab 2014;99:1915-42.

7. Corssmit EP, Romijn JA. Clinical management of paragangliomas. Eur J Endocrinol 2014;171:R231-43.

8. Hanmayyagari B, Voleti SN, Guntaka M, et al. An uncommon cause of hypertension: Paraganglioma revisited. CHRISMED J Health Res 2015;2:268-71.

9. Carty S, Young WF, Lin D. Paragangliomas: treatment of locoregional disease. Post TW, ed. UpToDate. Waltham, MA: UpToDate Inc.; 2018.

10. Waguespack SG, Rich T, Grubbs E, et al. A current review of the etiology, diagnosis, and treatment of pediatric pheochromocytoma and paraganglioma. J Clin Endocrinol Metab 2010;95:2023-37.

11. Dias Pérez JA, Currás Freixes M. Chromogranin A and neuroendocrine tumors. Endocrinol Nutr 2013;60:386-95.

12. Firoz T, Magee LA. Acute myocardial infarction in the obstetric patient. Obstet Med 2012;5:50-7.

13. Ryshten N, Janssens L, Ector B. Acute myocardial infarction due to spontaneous postpartum multivessel coronary artery dissection. J Cardiol Cases 2014;9:80-3.

14. Vanderveen KA, Thompson SM, Callstrom MR, et al. Biopsy of pheochromocytomas and paragangliomas: potential for disaster. Surgery 2009;146:1158-66.

15. Ahmad T, Patil S, Pasarad AK, et al. Paraganglioma masquerading as acute myocardial infarction and cardiogenic shock. Indian Heart J 2016;68:S258-63.

16. Satendra M, de Jesus C, Bordalo e Sá AL, et al. Reversible catecholamine-induced cardiomyopathy due to pheochromocytoma: a case report. Rev Port Cardiol 2014;33:177.e1-6.

17. Sibal L, Jovanovic A, Agarwal SC, Peaston RT, et al. Phaeochromocytomas presenting as acute crises after beta blockade therapy. Clin Endocrinol 2006;65:186-90. 